\title{
Regulation of Sticholysin II-Induced Pore Formation by Lipid Bilayer Composition, Phase State, and Interfacial Properties
}

Juan Palacios-Ortega, 1,2 Sara García-Linares, ,,2 Mia Åstrand,2 Md. Abdullah Al Sazzad, ${ }^{2}$ José G. Gavilanes, ${ }^{1}$ Álvaro Martínez-del-Pozo, ${ }^{1}$ and J.Peter Slotte ${ }^{2 *}$

${ }^{1}$ Departamento de Bioquímica y Biología Molecular I, Universidad Complutense, Madrid, Spain, and '2Biochemistry, Faculty of Science and Engineering, Åbo Akademi University, Turku, Finland

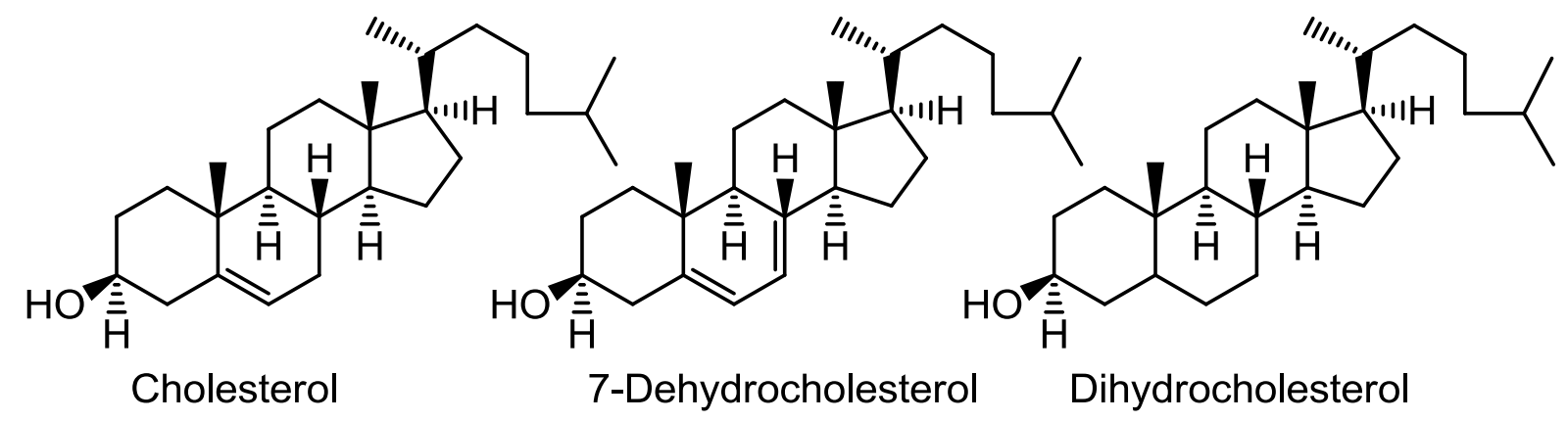

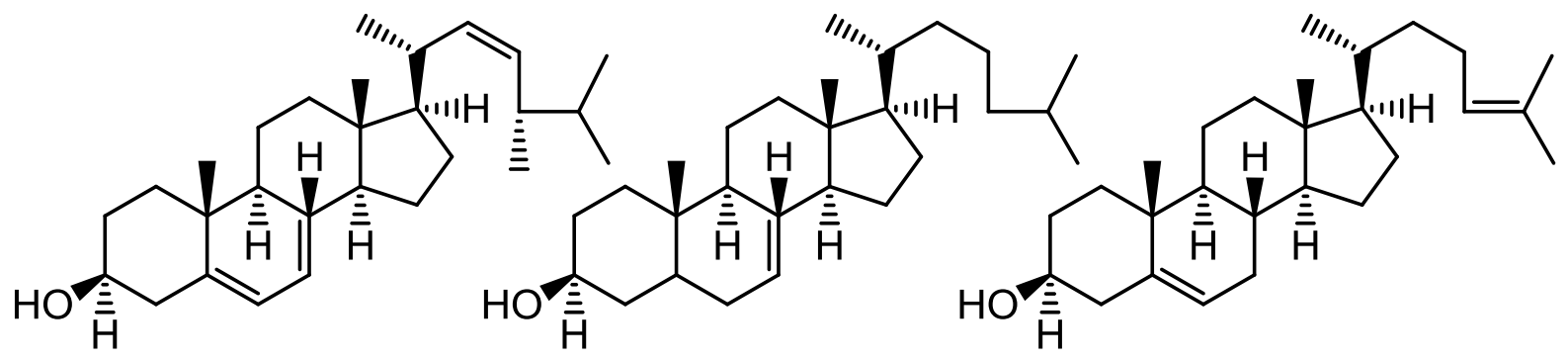

Ergosterol Lathosterol Desmosterol

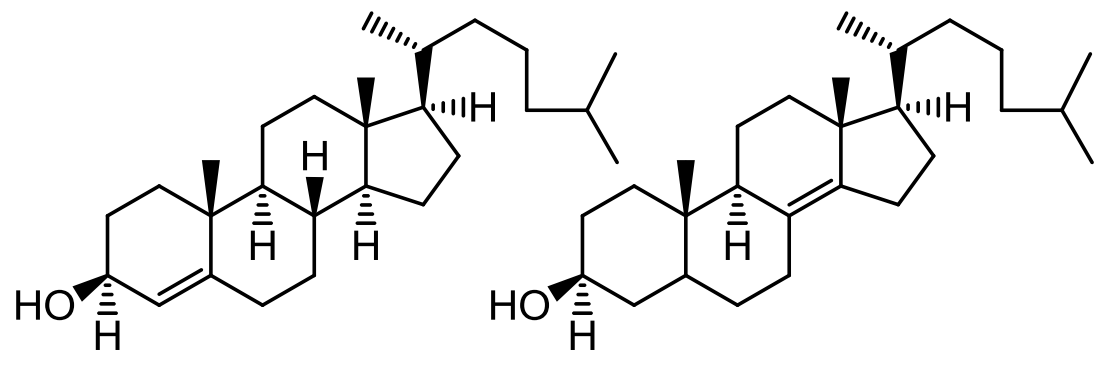

Allocholesterol 8(14)-Cholesten-3 3 -ol 

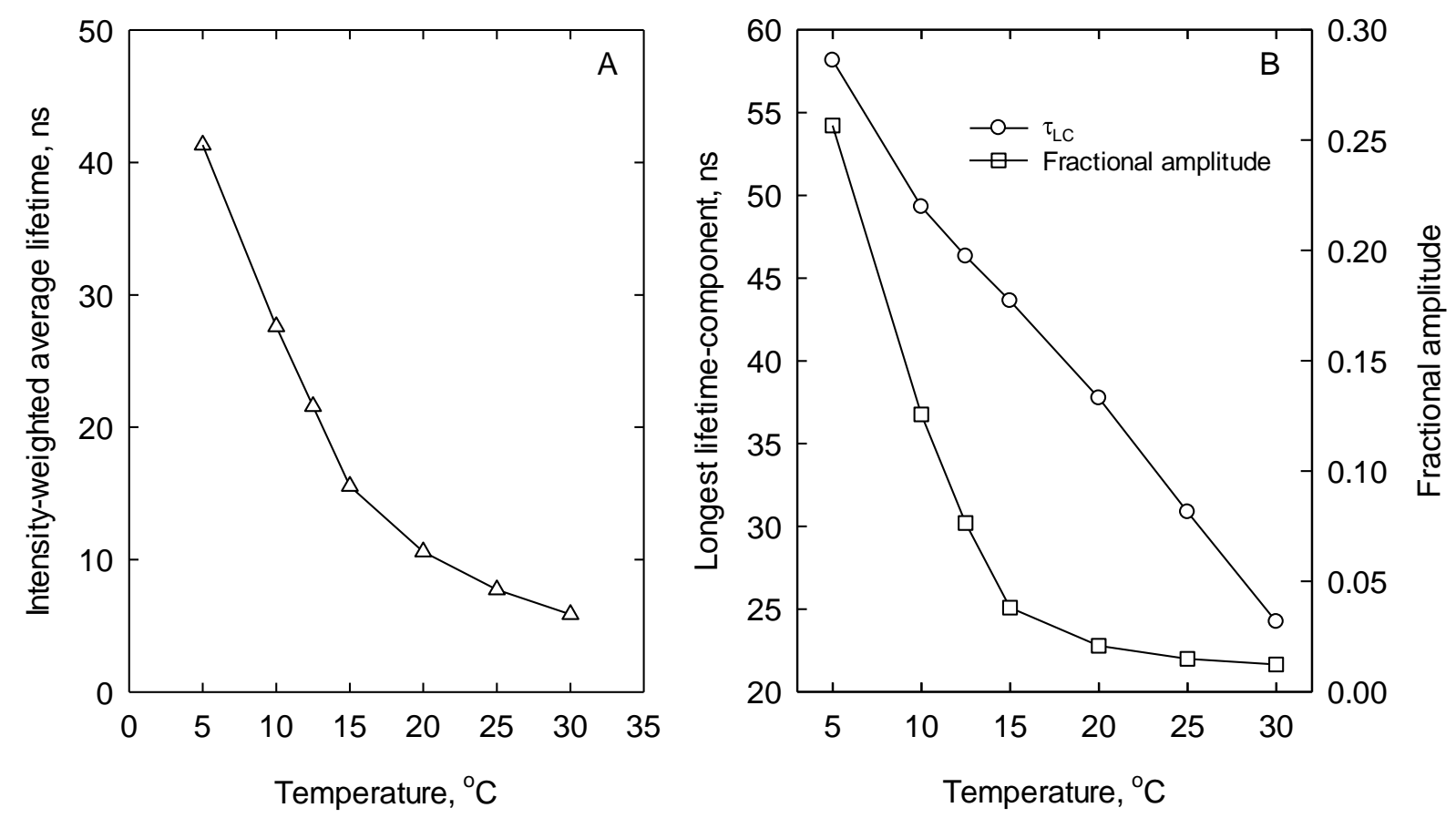

Figure S2. Fluorescence lifetime analysis of tPA in LUVs prepared from POPC and PSM (4:1 molar ratio). The LUVs were kept at the indicated temperature, and lifetime analysis was performed. Panel A shows intensity-weighted average lifetimes of TPA as a function of temperature. Panel B shown both the longest lifetime component of tPA fluorescence (open circles), and the fractional amplitude of that lifetime component (open squares), as a function of temperature. 